\title{
Investigating the effects of proton exchange membrane fuel cell conditions on carbon supported platinum electrocatalyst composition and performance
}

\author{
Anant Patel, Kateryna Artyushkova, ${ }^{\text {a) }}$ and Plamen Atanassov \\ Chemical and Nuclear Engineering Department, UNM Center for Emerging Energy Technologies, \\ University of New Mexico, Albuquerque, New Mexico 87131 \\ Vesna Colbow, Monica Dutta, David Harvey, and Silvia Wessel \\ Ballard Power Systems, Burnaby British Columbia, V5J 5J8 Canada
}

(Received 17 January 2012; accepted 10 April 2012; published 26 April 2012)

\begin{abstract}
Changes that carbon-supported platinum electrocatalysts undergo in a proton exchange membrane fuel cell environment were simulated by ex situ heat treatment of catalyst powder samples at $150{ }^{\circ} \mathrm{C}$ and $100 \%$ relative humidity. In order to study modifications that are introduced to chemistry, morphology, and performance of electrocatalysts, XPS, HREELS and three-electrode rotating disk electrode experiments were performed. Before heat treatment, graphitic content varied by $20 \%$ among samples with different types of carbon supports, with distinct differences between bulk and surface compositions within each sample. Following the aging protocol, the bulk and surface chemistry of the samples were similar, with graphite content increasing or remaining constant and Pt-carbide decreasing for all samples. From the correlation of changes in chemical composition and losses in performance of the electrocatalysts, we conclude that relative distribution of Pt particles on graphitic and amorphous carbon is as important for electrocatalytic activity as the absolute amount of graphitic carbon present. (c) 2012 American Vacuum Society. [http://dx.doi.org/10.1116/1.4707153]
\end{abstract}

\section{INTRODUCTION}

Increasing competition for fossil fuel resources and a growing awareness of the impact of greenhouse gases on the global environment has led to the development of alternative, green energy sources. ${ }^{1}$ These green energies, such as solar or wind, provide power from nonpolluting and freely available resources, but generally suffer from application restrictions and low efficiencies. Conversely, polymer electrolyte membrane fuel cells (PEMFCs) are high efficiency devices capable of using multiple fuels ${ }^{2}$ for both stationary and automotive applications. ${ }^{1-5}$ PEMFCs are open systems that generate power by separating two halves of an electrochemical reaction, oxidation of hydrogen and reduction to oxygen to form water.

As shown in Fig. 1, hydrogen diffuses through the support material until it reaches the platinum catalyst on the anode. The hydrogen molecules are separated into their component protons and electrons; the protons pass through a selectively permeable membrane to the cathode, whereas the electrons move through an outside circuit. On the cathode, oxygen is reduced and combined with the protons and electrons to form water, which then exits the cell. Though PEMFCs do have advantages over other alternative energy sources, they suffer from high overall cost and shortened lifespans. ${ }^{6-9}$ Platinum is the most common catalyst used in these devices, ${ }^{1,10-12}$ but increasing economic pressures are restricting its use. This has led to the current catalyst design of platinum nanoparticles dispersed on highly structured porous support material, ${ }^{7,13}$ generally carbon blacks

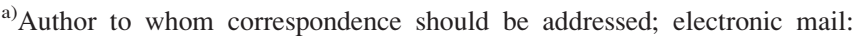
kartyush@unm.edu
}

(CB). ${ }^{14,15}$ Carbon blacks are used in fuel cells as electrocatalyst supports to ensure electronic conductivity between the electrocatalyst and the current collector. CBs also influence mass and heat transport providing a path for even gas distribution, for gas access to the electrocatalyst, and for the removal of products (gases and liquids). Surface chemistry and structural morphology are critical for mass transport properties, water and heat management, and subsequently electrocatalytic activity. Carbon blacks; however, lack the robustness of platinum electrodes ${ }^{1,15}$ and are susceptible to oxidation in the fuel cell environment. ${ }^{16,17}$ Oxidation of the support material can greatly reduce the porosity of the electrode, ${ }^{6,8,18}$ restricting the gas flow to the platinum catalyst, limiting the total power supplied by the cell. ${ }^{14,18,19}$ Carbon blacks are also highly diverse; chemical, morphological, and structural differences can change drastically between samples depending on the synthesis method. ${ }^{20}$ Although some factors, such as surface area and graphite content, have been shown to impact the durability of support materials, it is currently unclear as to what other variables promote or inhibit corrosion, ${ }^{8,14-16,21,22}$ making understanding the sample as a whole highly important.

Before PEMFCs can be considered commercially viable, support corrosion and performance losses need to be well understood. As such, the object of this work was to identify which properties of carbon blacks change when Pt-based electrocatalysts supported on these carbon blacks are exposed to the PEMFC environment. It has long been recognized that morphology, structure and surface properties of carbon materials strongly affect their tendency toward electrochemical corrosion. Platinum electrocatalysts prepared on several types of carbon black supports were subjected to a simulated PEMFC-like environment and were characterized 


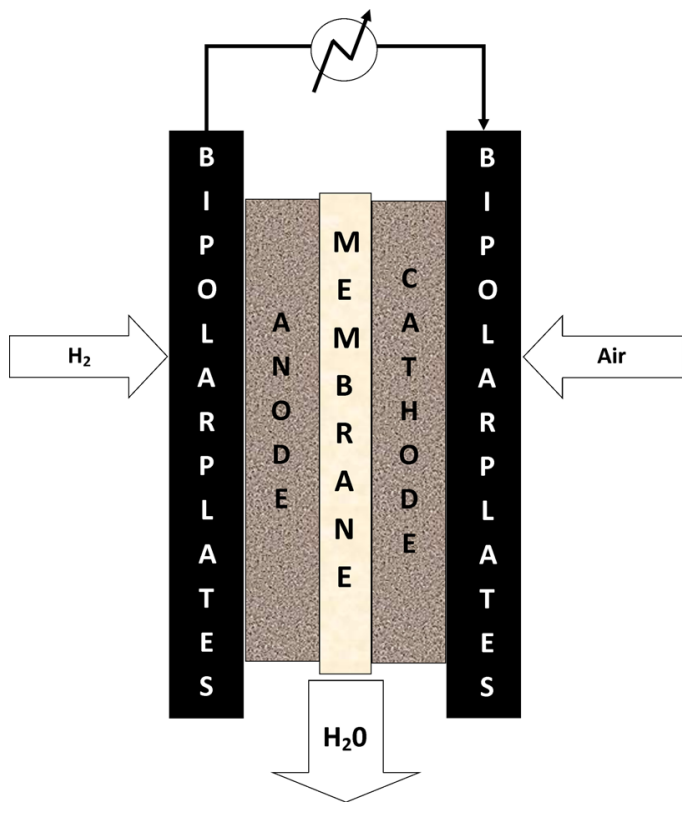

Fig. 1. (Color online) Simplified schematic of a hydrogen fuel cell; hydrogen is oxidized on the anode to protons and electrons, oxygen reduced on the cathode to form water.

before and after aging. As such, high-resolution electron energy-loss spectroscopy (HREELS) and x-ray photoelectron spectroscopy (XPS) were chosen to obtain information on graphitic content (degree of crystallinity) and surface oxide content of the carbon supports. Finally, rotating disk electrode (RDE) experiments were done to test the electrocatalytic performance of materials.

\section{MATERIALS AND METHODS}

\section{A. Materials}

In order to understand the effect of the type of carbon support on catalyst degradation, Pt catalysts on distinctly different carbon supports, low surface area (LSA), mid-range surface area (MSA), high surface area (HSA) carbons and, in addition, heat-treated high surface area carbon (HSAHT) Pt catalyst, each with 50 wt. \% Pt, were investigated. HSA carbons have a larger number of catalyst anchor sites yielding well-dispersed catalyst particles on the support. This level of dispersal is believed to result in increased kinetic performance. However, the large number of surface sites means these carbons will be more susceptible to corrosion; thus altering their performance and level of degradation. In order to stabilize the high surface area carbon Pt catalyst was heattreated to cause some degree of graphitization. Catalysts were aged by heating the powder samples for $2 \mathrm{~h}$ at $150{ }^{\circ} \mathrm{C}$ and $100 \%$ relative humidity (RH). Aged samples were analyzed as is by XPS, HREELS, and RDE experiments.

\section{B. Characterization}

XPS was performed using Kratos Axis Ultra spectrometer using an $\mathrm{Al} K \alpha \mathrm{x}$-ray source, with emission voltage of $12 \mathrm{kV}$ and emission current of $20 \mathrm{~mA}$. Both low resolution survey spectra and high resolution $\mathrm{C} 1 s$ and $\mathrm{Pt} 4 f$ spectra were acquired. Au powder was placed on each sample and $\mathrm{Au} 4 f$ spectra were acquired. All XPS spectra from each sample were charge-referenced to $\mathrm{Au} 4 f$ at $84 \mathrm{eV}$.

HREELS was done using a JEOL 2010F TEM using a field emission gun as the source with a voltage of $200 \mathrm{kV}$, and beam energy of $0.1 \mathrm{kV}$. The as-received and aged catalysts powders were investigated using this method. To ensure that the results were accurate, the spectra were acquired at 15 random locations per sample. Finally, highly ordered pyrolytic graphite (HOPG) was used as a reference sample because of its $100 \% s p^{2}$ bonding nature. Aberration corrected STEM images were acquired at JOEL 2200FS microscope at SHaRE user facility at Oak Ridge National Laboratory.

\section{Performance measurements}

A portion of the as-received and aged samples were weighed and added to a solution of $80 / 20$ de-ionized (DI) water/isopropyl alcohol and a $0.5 \%$ Nafion in DI water. The mixture was then sonicated to form a homogenous $4 \mathrm{mg} / \mathrm{mL}$ ink with $30 \mu \mathrm{L}$ of the Nafion solution per milligram of sample. To test the samples, $10 \mu \mathrm{L}$ of the catalyst ink were pipetted onto a rotating disk electrode, and was then dried for $30 \mathrm{~min}$ at $65^{\circ} \mathrm{C}$.

Using a rotation speed of $1600 \mathrm{rpm}$ and $0.1 \mathrm{M}$ perchloric acid as the electrolyte, the electrode was cycled between 0 and $1.2 \mathrm{~V}$ [versus reference hydrogen electrode (RHE)] 50 times at a rate of $100 \mathrm{mV} / \mathrm{s}$. This step was done to ensure a clean, uncontaminated surface. After the cleaning sweeps, oxygen gas was bubbled through the cell, and the electrode was cycled between 0 and $1.2 \mathrm{~V}$ (versus RHE) at a rate of $5 \mathrm{mV} / \mathrm{s}$. The exchange current densities and apparent Tafel slopes for oxygen reduction reaction were then extracted from these curves to quantify performance losses due to aging. The exchange current density and apparent Tafel slopes were calculated by plotting the first $100 \mathrm{mV}$ of the cathodic sweep below the open circuit potential on a semilog plot and fitting a linear trendline to the curve. The slope of the trendline would be equivalent to the apparent Tafel slope, whereas the intercept would be equal to the exchange current density.

\section{RESULTS AND DISCUSSION}

Figure 2 shows representative high resolution dark field ac STEM images from as-received electrocatalyst powders on four types of carbon support. Significant agglomeration of particles is evident for LSA50 catalyst sample. Similar size and dispersion of particles are present for MSA50 and HSA50 catalysts. However, the large number of surface sites means these carbons will be more susceptible to corrosion; thus, altering their performance and level of degradation. In order to stabilize the high surface area carbon catalyst, it was heat-treated to cause some degree of graphitization. Larger $\mathrm{Pt}$ particles are evident from ac STEM micrographs for HSAHT50 sample.

The ability to discriminate between different carbon chemical environments, not just elemental compositions, is one of the primary advantages of XPS in the characterization 

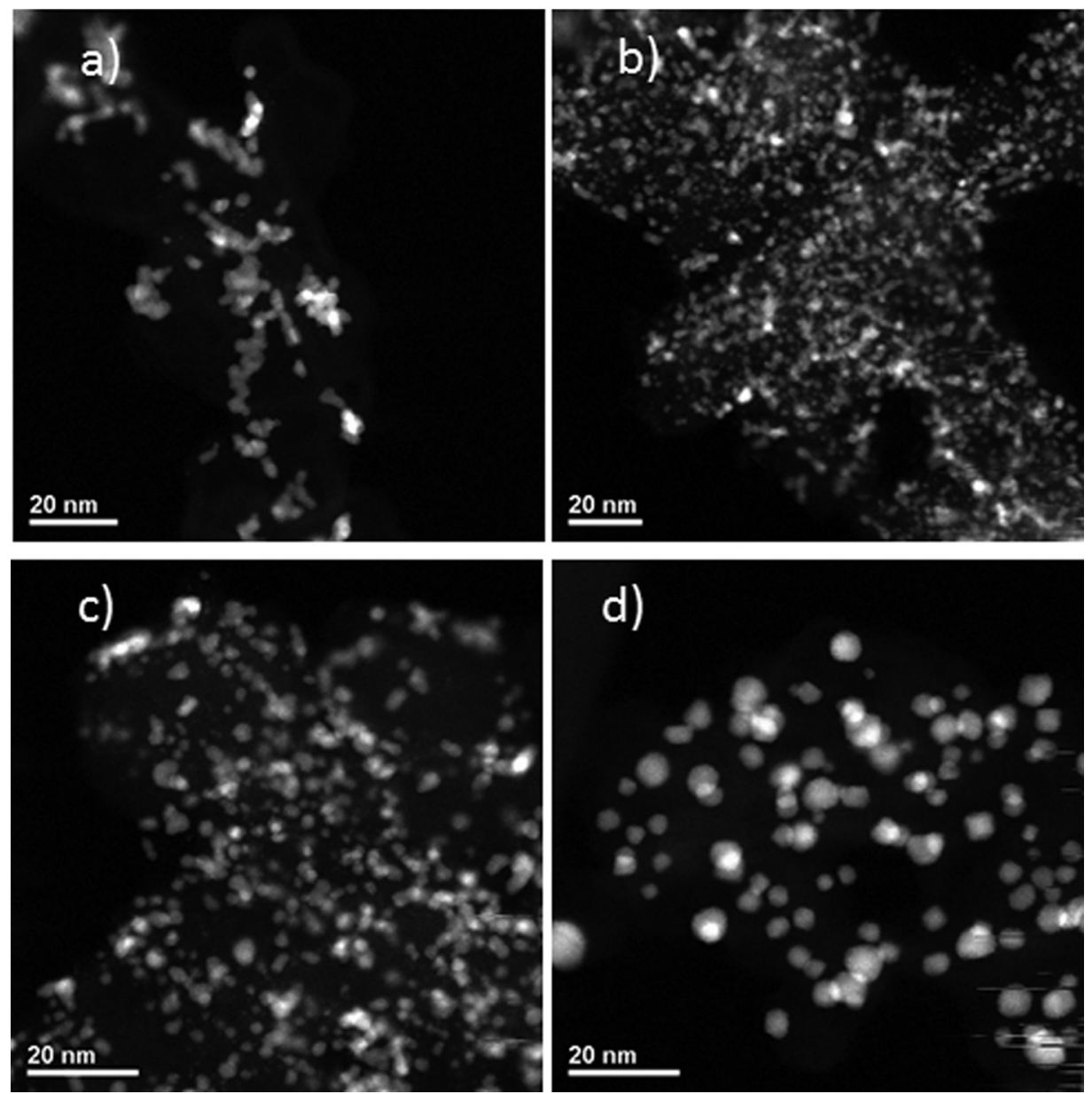

FIG. 2. AC STEM dark field images of electrocatalysts (a) LSA 50, (b) MSA 50, (c) HSA 50, and (d) HSAHT 50

of carbon corrosion; however, HREELS is the preferred method to quantitatively extract the $s p^{2}$ fraction of carbonbased materials from carbon $K$-edge spectrum.

\section{A. XPS results}

Figure 3 shows C $1 s$ XPS spectra for LSA50 and MSA50 samples along with curve fit. A different ratio of graphitic peak at $284.5 \mathrm{eV}$ to the rest of the peak is obvious. Table I shows results of curve fit of XPS spectra for as-received and aged samples. The values shown are the average of three areas.

For as-received samples, increase in surface area anticorrelates with graphitic content, with MSA50 having the lowest percentage of graphitic carbon. Samples with lowest graphitic content also have the highest amounts of aliphatic carbons. A heat-treated HSAHT50 sample has the same amount of surface oxides, but there is a reorganization of graphitic and aliphatic carbons. Heat treatment of HSA50 catalyst increases graphite content to almost the same level as in the LSA50 sample.

Aging of LSA50 and HSAHT50 catalyst powders cause insignificant changes in the amount of graphitic carbon. The largest changes are detected for the MSA50 catalyst where an $\sim 30 \%$ increase of graphitic content is observed and HSA50 sample with an $\sim 25 \%$ increase. For both of these samples, decrease is amorphous aliphatic carbon is detected. Additionally, the relative amount of surface oxides increases with aging even though the total amount of oxygen is decreased by $\sim 50 \%$ for all samples.

The total platinum content detected by XPS, as detailed in Table II, increased for all four samples with aging. Due to surface sensitivity of XPS sampling depth, this result might point toward significant segregation of Pt toward top layers upon heat treatment. Similar amounts of platinum were found for all samples after aging.

Figure 4 and Table III show results of curve fitting Pt $4 f$ spectra for as-received and aged samples. In as-received catalyst samples, an increase of surface area causes an increase in relative amount of metallic Pt. The heat-treated HSAHT50 catalyst sample has even a larger degree of $\mathrm{Pt}$ reduction.

After aging, all catalyst samples result in a very similar composition with $\sim 40 \%$ of metallic Pt and $30 \%$ of oxides. An increase in a metallic type of Pt is observed with decreasing surface area of the catalyst supports. LSA50 and HSA50 catalysts have large increase in oxides, whereas unchanged for the MSA50 catalyst. For the HSAHT50 sample, the 

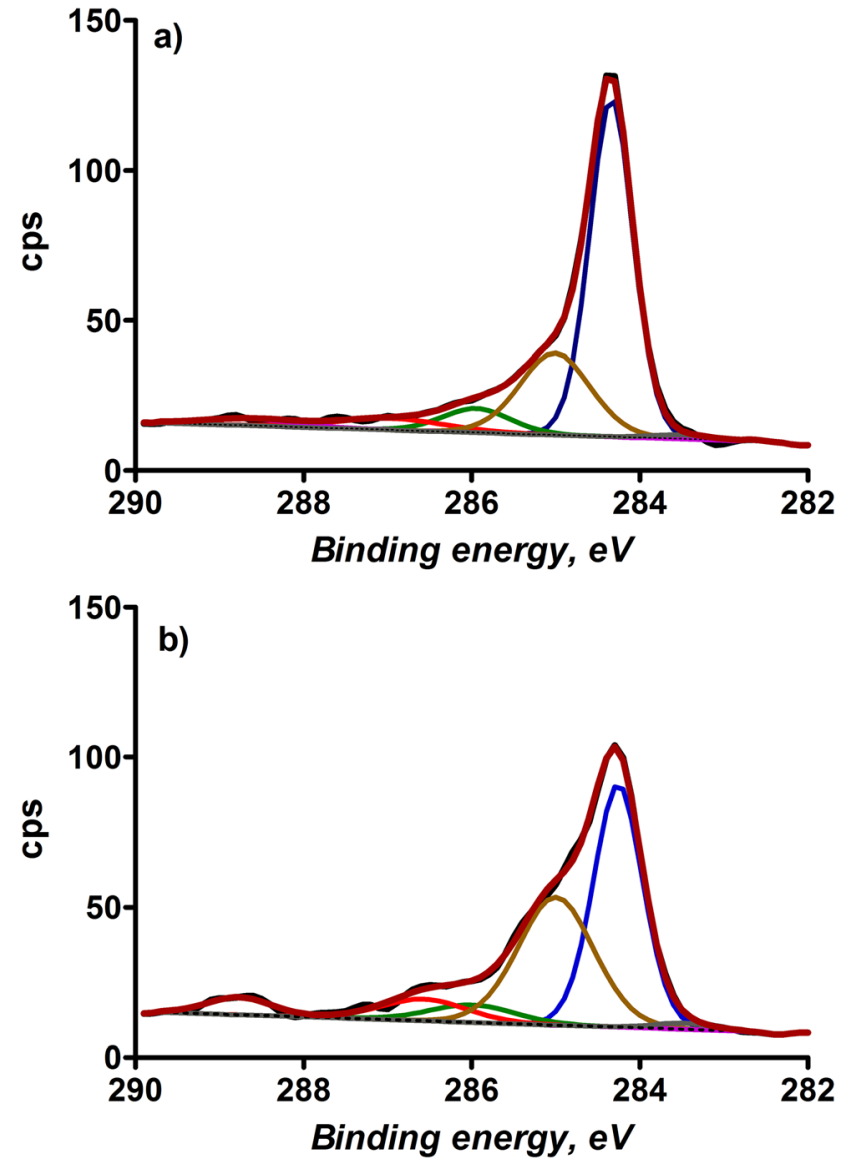

FIG. 3. (Color online) XPS high resolution C $1 s$ spectra for (a) LSA50 and (b) MSA50 samples shown with curve fits used to deconvolve species as identified in Table I.

largest formation of oxides is observed and 30\%-40\% loss of Pt bound to carbon is observed for all samples, which correlates with the loss of amorphous carbon detected by $\mathrm{C} 1 \mathrm{~s}$ spectra.

To summarize, XPS shows that for the LSA50 catalyst, heat treatment introduces no changes in carbon speciation, but significantly increases relative metallic and platinum oxide content with accompanying loss of $\mathrm{Pt}-\mathrm{C}$. The MSA50 catalyst displays large changes in both carbon and Pt compo-

TABLE I. C speciation as determined from XPS spectra.

\begin{tabular}{lccccc}
\hline \hline Sample & Cgraphitic & $\mathrm{C}-\mathrm{C}$ & $\mathrm{C} * \mathrm{C}-\mathrm{X}$ & $\mathrm{C}-\mathrm{OH} / \mathrm{C}-\mathrm{OC}$ & $\mathrm{C}=\mathrm{O}$ \\
\hline $\begin{array}{l}\text { Binding } \\
\text { energy (eV) }\end{array}$ & 284.5 & 285.1 & 285.7 & 286.7 & 289.1 \\
& \multicolumn{6}{c}{ As-received } \\
LSA50 & 59.8 & 25.4 & 6.1 & 5.7 & 2.9 \\
MSA50 & 40.2 & 41.0 & 8.6 & 5.9 & 4.4 \\
HSA50 & 45.6 & 35.8 & 6.9 & 7.0 & 4.7 \\
HSAHT50 & 57.7 & 26.4 & 3.8 & 8.6 & 3.5 \\
& & & & & \\
LSA50 & 59.7 & 21.4 & 6.1 & 9.4 & 3.4 \\
MSA50 & 56.6 & 23.0 & 8.1 & 8.7 & 3.6 \\
HSA50 & 56.4 & 23.5 & 5.6 & 11.8 & 2.8 \\
HSAHT50 & 53.4 & 25.8 & 6.9 & 9.4 & 4.6 \\
\hline \hline
\end{tabular}

TABLE II. Platinum surface content comparison as calculated from XPS.

\begin{tabular}{lcc}
\hline \hline Sample & Pt \% as-received & Pt \% aged \\
\hline LSA50 & 4.3 & 6.6 \\
MSA50 & 2.8 & 7.5 \\
HSA50 & 2.5 & 6.1 \\
HSAHT50 & 1.9 & 4.7 \\
\hline \hline
\end{tabular}

sitions. The HSA50 catalyst shows no changes in metallic Pt percentage, but a very significant loss of amorphous carbon and Pt bound to $\mathrm{C}$. The HSAHT50 catalyst has the smallest changes detected in carbon but significant loss of metallic $\mathrm{Pt}$ and increase in $\mathrm{PtO}$.

An increase in relative amounts of graphitic carbon and metallic platinum after ex situ treatment is mainly due to the loss of amorphous carbon and associated Pt. There is an expected increase of $\mathrm{Pt}$ oxides during heat treatment in oxidizing atmosphere; however, the largest changes are in the increase of metallic Pt. As XPS data reported are in relative atomic percent, decrease in the amount of one type of species leads to relative increase of the other types of species. Therefore, an increase in metallic $\mathrm{Pt}$ and graphitic $\mathrm{C}$ may mainly be due to the loss of $\mathrm{P}$ associated with amorphous carbon. Thus, the major results of aging of electrocatalysts is a loss of amorphous carbon, accompanied by a loss of $\mathrm{Pt}$
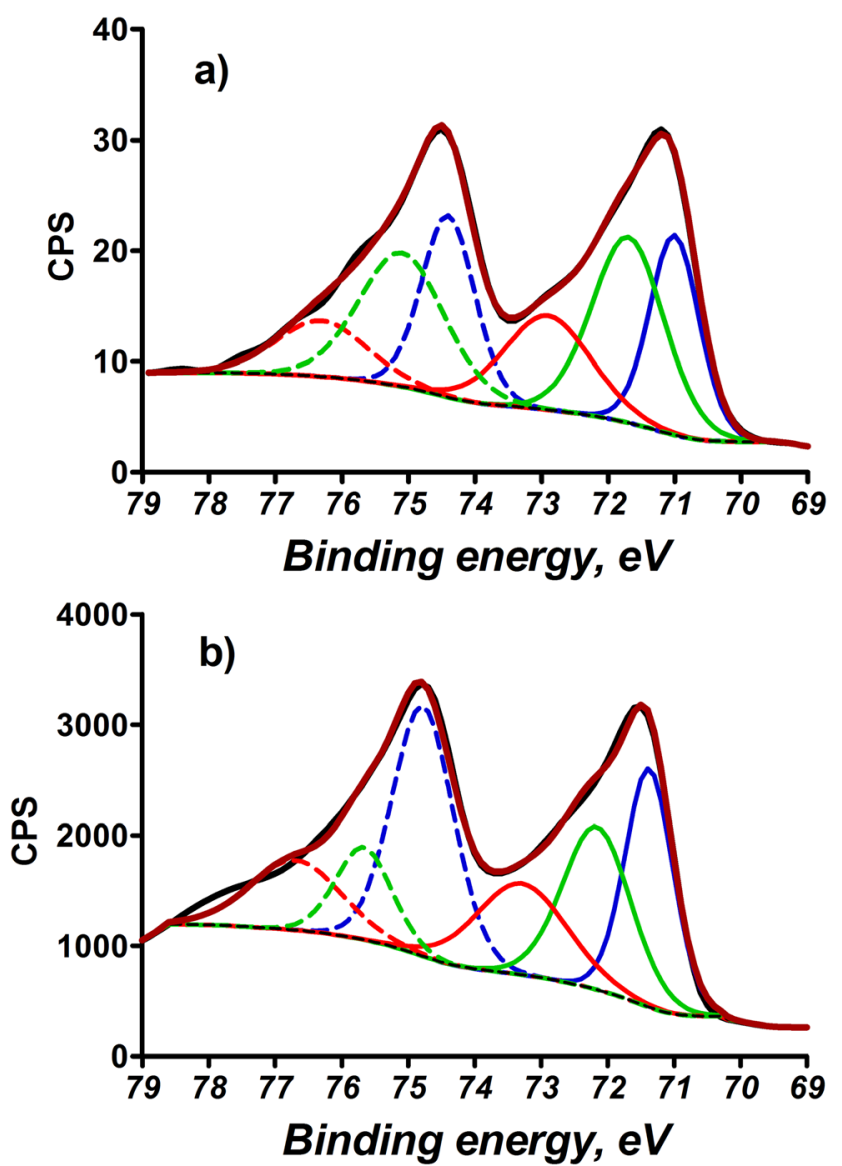

FIG. 4. (Color online) Platinum speciation for LSA50 before (a) and after $e x$ situ aging (b). 
TABLE III. Pt speciation as determined from XPS spectra.

\begin{tabular}{|c|c|c|c|}
\hline Sample & $\mathrm{Pt}$ & $\mathrm{Pt} / \mathrm{Pt}-\mathrm{C}_{x} \mathrm{H}_{y}$ & $\mathrm{PtO} / \mathrm{Pt}(\mathrm{OH}) 2$ \\
\hline Binding energy $(\mathrm{eV})$ & 71.1 & 71.7 & 72.8 \\
\hline \multicolumn{4}{|c|}{ As-received } \\
\hline LSA50 & 30.4 & 45.7 & 23.9 \\
\hline MSA50 & 33.5 & 36.2 & 30.2 \\
\hline HSA50 & 40.9 & 34.0 & 25.1 \\
\hline HSAHT50 & 59.1 & 32.8 & 8.1 \\
\hline \multicolumn{4}{|c|}{ Aged } \\
\hline LSA50 & 40.0 & 26.8 & 33.2 \\
\hline MSA50 & 42.0 & 26.8 & 31.2 \\
\hline HSA50 & 41.3 & 22.0 & 36.6 \\
\hline HSAHT50 & 47.4 & 23.7 & 29.0 \\
\hline
\end{tabular}

bound to it and simultaneous segregation at the very top surface of metallic Pt particles covered by Pt oxide.

\section{B. HREELS results}

From the higher energy in the HREELS spectrum, excitations arising from transitions of inner-shell electrons to unoccupied states above the Fermi level can be obtained. For carbon, the $K$-edge spectrum represents the transition from the $1 s$ core level to the unoccupied anti-bonding state in the $2 p$ energy level, $\sigma^{*}$ and $\pi^{*}$. Direct measure of $\sigma^{*}$ and $\pi^{*}$ states of carbonaceous materials allows accurate measure-
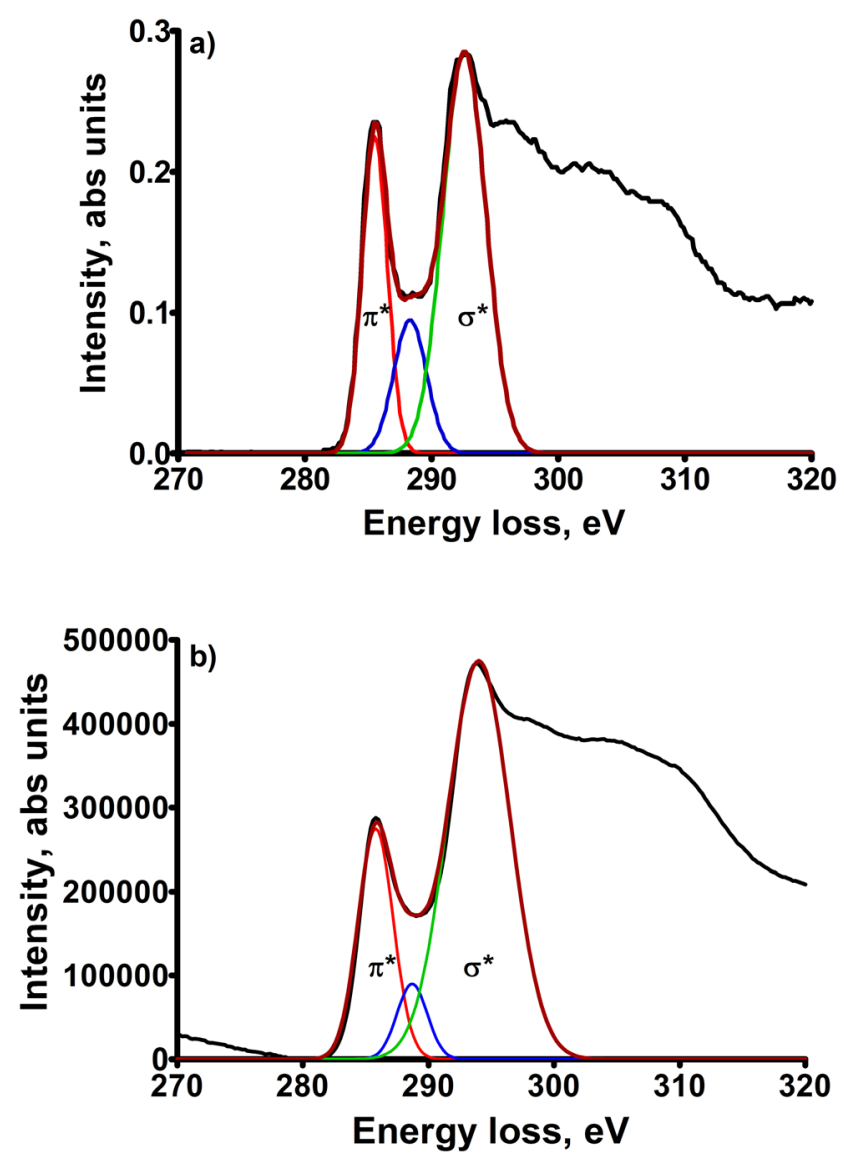

FIG. 5. (Color online) HREELS spectra for (a) HPOG and (b) LSA50 catalyst shown with curve fits used to deconvolve $\sigma^{*}+\pi^{*}$ contributions.
TABLE IV. $s p^{2}$ Percentage determined from HREELS spectra.

\begin{tabular}{lccc}
\hline \hline Sample & As-received $(\%)$ & HT $(\%)$ & \% Increase \\
\hline LSA50 & 78.7 & 85.0 & 8.0 \\
MSA50 & 69.7 & 79.2 & 13.6 \\
HSA50 & 59.4 & 77.3 & 30.0 \\
HSAHT50 & 80.2 & 83.6 & 4.2 \\
\hline \hline
\end{tabular}

ment of $s p^{2}$ and $s p^{3}$ states provided a suitable reference material with $100 \% s p^{2}$ hybridization. For both unknown and reference materials, HREELS $K$-edge spectra can be used to calculate respective integrated areas under each energy loss peak at $K$-edge, from which the $s p^{2}$ fraction can be calculated as

$$
s p^{2} \%=\frac{A_{p *}}{A_{p *+s *}} / \frac{R_{p *}}{R_{p *+s *}},
$$

where $A$ is sample, and $R$ is $100 \% s p^{2}$ bonded reference material, the subscripts $\pi^{*}$ and $\left(\sigma^{*}+\pi^{*}\right)$ represent integrated areas under each energy loss peak at $K$-edge determined by a curve fitting procedure as described in the following. ${ }^{23,24}$

HOPG was used as a standard for $100 \% s p^{2}$ hybridized reference material. The spectra were curve fitted with three Gaussian curves to determine areas under the peaks used for quantifying the ratio of $s p^{2}$ to $s p^{3}$ bonds. Figure 5 shows HREELS spectra for HOPG and LSA sample with three peaks used for curve fitting. The $\pi^{*}$ feature is present at an energy loss of $\sim 285 \mathrm{eV}$, which represents a transition of $\mathrm{C}$ $1 s$ electrons to unoccupied $\pi^{*}$ state, whereas the $\sigma^{*}$ feature at $291 \mathrm{eV}$ reflects transition to unoccupied $\sigma^{*}$ state. The $\pi^{*}$ feature is typical for an $s p^{2}$ hybridized carbon. The percent of $s p^{2}$ bonded carbon then can be calculated using Eq. (1).

Table IV shows the percent of $s p^{2}$ carbon determined from HREELS spectra for four samples as-received and after

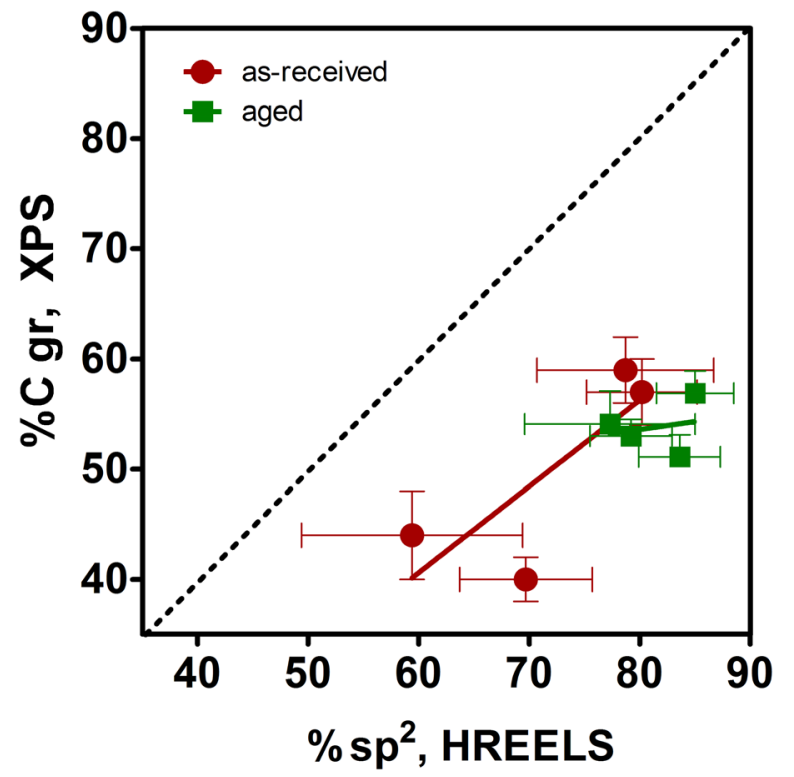

FIG. 6. (Color online) \% Graphitic carbon as determined by XPS vs $\% s p^{2}$ content as determined by HREELS. 
TABLE V. Apparent Tafel slopes ( $\mathrm{mV} /$ decade) derived from RDE experiments.

\begin{tabular}{lccc}
\hline \hline Sample & As-received & After aging & \% Change \\
\hline LSA50 & 27.2 & 21.5 & -20.8 \\
MSA50 & 30.6 & 27.5 & -10.3 \\
HSA50 & 32.8 & 29.1 & -11.3 \\
HSAHT50 & 24.0 & 28.3 & +18.0 \\
\hline \hline
\end{tabular}

ex situ aging treatment at $150{ }^{\circ} \mathrm{C}$ in air $100 \%$ humidity. Catalysts have very different levels of graphitic content with the highest for LSA50 and HSAHT50 catalyst samples at $\sim 80 \%$. With increasing surface area, the graphitic content decreases up to $60 \%$ for HSA50 catalyst. Aging samples at $150{ }^{\circ} \mathrm{C}$ in $100 \% \mathrm{RH}$ results in samples with quite similar levels of graphite content ranging from $77 \%$ to $85 \%$. The trend in relative graphitic content for aged samples is the same as for as-received samples, but the range of it is smaller.

The trend in change of graphitic content determined by HREELS is very similar to that detected by XPS, except for the MSA50 catalyst having a smaller relative percent of graphitic carbon as detected by XPS then the HSA50 catalyst. This might be due to the surface sensitivity of XPS in comparison with HREELS, pointing toward larger relative amounts of surface carbon oxides.

Figure 6 plots percent of graphitic content determined by XPS versus that determined by HREELS for both asreceived and aged samples. A good correlation is observed for both sets of samples. For as-received samples, a smaller spread of values detected by XPS indicates that the surface is different from the bulk and surfaces of all catalysts are more similar to each other than the bulk of catalysts. Aged samples are very similar to each other as detected by both methods, but again, similarity is larger at the very top $10 \mathrm{~nm}$ as seen by XPS. The graphitic carbon content of all samples after aging is similar to that of the untreated LSA50 and the HSAHT50 catalyst samples.

\section{RDE results}

Catalyst performance losses were quantified using rotating disk electrode experiments. Two sets of catalysts were tested for this study: as-received catalysts and ex situ aged catalysts. The apparent Tafel slopes calculated and exchanged current densities for as-received and heat-treated samples as well as percent change in them are shown in Tables V and VI.

TABLE VI. Exchange current densities $\left(\mathrm{mA} / \mathrm{cm}^{2}\right)$ derived from RDE experiments.

\begin{tabular}{lccc}
\hline \hline Sample & As-received & After aging & \% Change \\
\hline LSA50 & 4.2 & 2.9 & -32.2 \\
MSA50 & 3.8 & 3.2 & -17.2 \\
HSA50 & 4.0 & 3.9 & -1.6 \\
HSAHT50 & 3.1 & 3.4 & +9.9 \\
\hline \hline
\end{tabular}

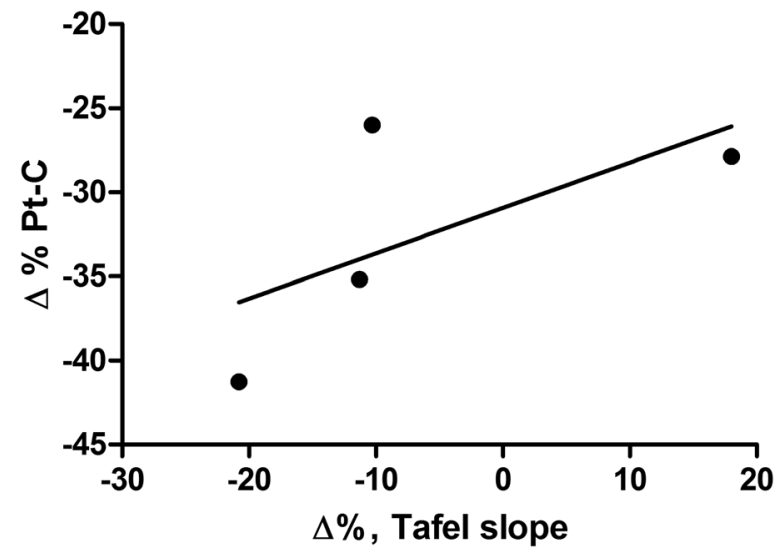

FIg. 7. Changes in apparent Tafel slopes vs relative Pt-C content on the surface.

The data shows that through the ex situ aging, the performance of all catalysts is changed, but there is no specific trend with types of carbon support. Although XPS and HREELS data showed that the relative graphitic content and the amount of metallic Pt increases with aging, it generally decreased the overall performance of the catalysts. Except for HSAHT50, the loss of performance is correlated with an increase in graphitic content as detected by both XPS and HREELS.

Figure 7 plots the changes in percentages of $\mathrm{Pt}$ bound to carbon versus change in the apparent Tafel plot. A linear correlation between these two parameters is observed for all samples, indicating that the larger the loss of the $\mathrm{Pt}$ that is coordinated with carbon the larger the loss is in performance.

Hence, correlation of surface carbon and Pt speciation as determined by XPS with electrochemical performance indicates that an absolute amount of graphitic content is not the only parameter responsible for better stability. Relative distribution of Pt particles on graphitic and amorphous carbon is as responsible for the losses of electrocatalytical activity. After aging, the largest losses that resulted in the degraded performance are associated with losses of amorphous carbon and associated dispersed Pt.

Although decreased performance due to aging is a welldocumented phenomenon, seeing an increase in the apparent Tafel slope and exchange current density of HSAHT catalyst is unexpected. ${ }^{6,8,22}$ Aging of this sample caused the smallest changes to carbon chemistry and caused loss of metallic $\mathrm{Pt}$ with accompanying significant growth of PtO. We will further investigate how these changes may affect performance.

\section{CONCLUSIONS}

Ex situ heat treatment of four carbon supported platinum catalysts was performed in order to determine the effect graphitic content has on the catalyst performance. The samples were analyzed using XPS, HREELS, and RDE experiments. Both XPS and HREELS results showed that graphite and metallic platinum content increased due to the heat treatment, and that the surface of the samples differed from the bulk. In addition, it was shown that as-after aging catalysts displayed decreased performance. After aging, the largest losses that resulted in the degrading performance are 
associated with losses of amorphous carbon and associated $\mathrm{Pt}-\mathrm{C}$ species. Relative distribution of $\mathrm{Pt}$ particles on graphitic and amorphous carbon is thus a critical parameter responsible for electrocatalytical activity and stability.

\section{ACKNOWLEDGMENTS}

The authors acknowledge the financial assistance from the Department of Energy EERE Hydrogen and Fuel Cell Technology Program (Project DE-EE0000466). They also acknowledge SHaRE facility at ORNL for access to AC STEM microscope (user proposal 2011_Artyushkova_66).

${ }^{1}$ R. Borup et al., Chem. Rev. 107, 3904 (2007).

${ }^{2}$ E. Antolini, T. Lopes, and E. R. Gonzalez, J. Alloy Compd. 461, 253 (2008).

${ }^{3}$ X. Yu and S. Ye, J. Power Sources 172, 133 (2007).

${ }^{4}$ X. Yu and S. Ye, J. Power Sources 172, 145 (2007).

${ }^{5}$ K. S. Dhathathreyan, P. Sridhar, G. Sasikumar, K. K. Ghosh, G. Velayutham, N. Rajalakshmi, C. K. Subramaniam, M. Raja, and K. Ramya, Int. J. Hydrogen Energy 24, 1107 (1999).

${ }^{6}$ H. Chizawa, Y. Ogami, H. Naka, A. Matsunaga, N. Aoki, T. Aoki, and K. Tanaka, ECS Trans. 11, 981 (2007).

${ }^{7}$ M. S. Mamat, S. A. Grigoriev, K. A. Dzhus, D. M. Grant, and G. S. Walker, Int. J. Hydrogen Energy 35, 7580 (2010).

${ }^{8}$ C. G. Chung, L. Kim, Y. W. Sung, J. Lee, and J. S. Chung, Int. J. Hydrogen Energy 34, 8974 (2009).
${ }^{9}$ J. Zhang, B. A. Litteer, W. Gu, H. Liu, and H. A. Gasteiger, J. Electrochem. Soc. 154, B1006 (2007).

${ }^{10}$ K. A. Starz, E. Auer, T. Lehmann, and R. Zuber, J. Power Sources 84, 167 (1999).

${ }^{11}$ Y. Dai, L. Ou, W. Liang, F. Yang, Y. Liu, and S. Chen, J. Phys. Chem. C 115, 2162 (2011).

${ }^{12}$ M. Oezaslan and P. Strasser, J. Power Sources 196, 5240 (2011).

${ }^{13}$ F. Dundar, A. Smirnova, X. Dong, A. Ata, and N. Sammes, J. Fuel Cell Sci. Technol. 3, 428 (2006).

${ }^{14}$ F. Xu, M. Wang, Q. Liu, H. Sun, S. Simonson, N. Ogbeifun, E. Stach, and J. Xie, ECS Trans. 33, 1281 (2010).

${ }^{15}$ A. P. Young, J. Stumper, and E. Gyenge, J. Electrochem. Soc. 156, B913 (2009).

${ }^{16}$ M. L. Perry, T. Patterson, and C. Reiser, ECS Trans. 3, 783 (2006).

${ }^{17}$ W. R. R. Baumgartner, E. Wallnofer, T. Schaffer, V. Hacker, V. Peinecke, and P. Prenninger, ECS Trans. 3, 811 (2006).

${ }^{18}$ P. T. Yu, W. Gu, R. Makharia, F. T. Wagner, and H. A. Gasteiger, ECS Trans. 3, 797 (2006).

${ }^{19}$ P. J. Ferreira, G. J. la, O', Y. Shao-Horn, D. Morgan, R. Makharia, S. Kocha, and H. A. Gasteiger, J. Electrochem. Soc. 152, A2256 (2005).

${ }^{20} \mathrm{~J}$. Li, P. He, K. Wang, M. Davis, and S. Ye, ECS Trans. 3, 743 (2006).

${ }^{21}$ D. Liang, Q. Shen, M. Hou, Z. Shao, and B. Yi, J. Power Sources 194, 847 (2009).

${ }^{22}$ D. A. Stevens, M. T. Hicks, G. M. Haugen, and J. R. Dahn, J. Electrochem. Soc. 152, A2309 (2005).

${ }^{23}$ M. Knauer, M. E. Schuster, D. Su, R. Schlögl, R. Niessner, and N. P. Ivleva, J. Phys. Chem. A 113, 13871 (2009).

${ }^{24}$ S. R. P. Silva and V. Stolojan, Thin Solid Films 488, 283 (2005). 